\title{
The combined modulation of Jovian and Galactic electrons in the heliosphere
}

\author{
Marius S Potgieter ${ }^{1}$ \\ Centre for Space Research, North-West University, Potchefstroom, South Africa \\ E-mail: marius.potgieter@nwu.ac.za

\section{Rendani R. Nndanganeni} \\ Centre for Space Research, North-West University, Potchefstroom, South Africa \\ Now at: Space Sciences, South African Space Agency (SANSA), Hermanus, South Africa
}

\begin{abstract}
The propagation and modulation of electrons in the heliosphere play a significant role in improving our understanding and assessment of the competing processes of solar modulation. An in-house developed, full three-dimensional numerical model is used to study the total modulation of Jovian and Galactic electrons from $1 \mathrm{MeV}$ to $50 \mathrm{GeV}$, and from the heliopause into the heliosphere, up to Jupiter and the Earth. For this purpose the Jovian electron source and the heliopause (a very local interstellar) electron spectrum must be specified in the model. The latter can be computed with confidence since Voyager 1 already crossed the heliopause returning the measured spectra from this very local interstellar region. Modeling results are compared with Voyager 1 observations in the outer heliosphere, including the heliosheath, as well as observations at or near the Earth, in particular the 2009 spectrum from the PAMELA space mission, ISSE 3 and from Ulysses for 1991 and 1992. Making use of the observations at Earth and the source function for the Jovian electrons, the energy range over which the Jovian electrons dominate the Galactic electrons can be determined. The intensity of Galactic electrons at Earth is computed subsequently. This gives a conclusive finding about the energy range where the Jovian particles dominate the Galactic electrons and determining the absolute intensity value that the Galactic particles can have at the Earth. The highest differential intensity for the Galactic electrons at the Earth for kinetic energy of $1 \mathrm{MeV}$ is $\sim 20 \mathrm{~m}^{-2} \mathrm{~s}^{-1} \mathrm{sr}^{-1} \mathrm{MeV}^{-1}$, whereas for Jovian electrons it is $\sim 400 \mathrm{~m}^{-2} \mathrm{~s}^{-1} \mathrm{sr}^{-1} \mathrm{MeV}^{-1}$.
\end{abstract}

35th International Cosmic Ray Conference - ICRC2017

10-20 July, 2017

Bexco, Busan, Korea

\footnotetext{
${ }^{1}$ Speaker

(C) Copyright owned by the author(s) under the terms of the Creative Commons

Attribution-NonCommercial-NoDerivatives 4.0 International License (CC BY-NC-ND 4.0).
} 


\section{Introduction}

Modulation of cosmic ray (CR) electrons in the heliosphere plays an important role in improving our understanding and evaluation of the heliospheric processes responsible for solar modulation, and the behaviour of low-energy Jovian and Galactic electrons, in particular. Numerical modeling, applied to this topic, provides a useful and essential tool in understanding and estimating the propagation tensor and other modulation parameters applicable to heliospheric modulation; see the review by [1]. The transport and propagation of Galactic CRs in the heliosphere is essentially determined by four major processes: convection and adiabatic energy losses caused by the expanding solar wind; diffusion caused by irregularities in the global heliospheric magnetic field (HMF); particle drifts caused by gradients and curvatures in the HMF and by the wavy heliospheric current sheet (HCS), with its tilt angle as solar activity proxy. All these processes are important for the modulation of Jovian and Galactic electrons. However, electrons experience far less adiabatic energy losses than protons and as such they respond directly to changes of the main modulating parameters, in particular the three diffusion coefficients (DCs), down to very low energies (kinetic energy $E \sim 1 \mathrm{MeV}$ ).

Numerical modeling requires in the first place that an input spectrum must be specified at an assumed heliospheric modulation boundary. The heliopause (HP) is considered such a boundary, where this input spectrum is assumed to be the very local interstellar spectrum (VLIS). The modulated Galactic electron spectra presented here are based on a newly established VLIS, or heliopause spectrum (HPS), determined from the knowledge when Voyager 1 (V1) observed and crossed the HP around 122 AU [2]. The Jovian electron source (JES) function at $5 \mathrm{AU}$ must also be constructed and is done using Ulysses, Pioneer 10, and other observations that was made in the vicinity of Jupiter, and related to observations at the Earth [see e.g. 3,4.5, and references there-in].

The main purpose of this work is to illustrate the overall combined modulation of Jovian and Galactic electrons in the heliosphere, using new knowledge of the location of the HP, the HPS and the JES in a comprehensive 3D drift-model. Modulated electron spectra for Jovian and Galactic electrons are computed, first separately and then together as a combined total electron spectrum at the Earth. Based on these efforts, the unknown intensity of Galactic electrons below $50 \mathrm{MeV}$ at the Earth is estimated.

\section{Numerical model}

A full three-dimensional (3D) numerical model is used to compute electron spectra at selected positions in the heliosphere. This particular model, all the DCs, the drift coefficient and other modulation parameters such as the solar wind speed and profile, and the HMF, are described in great detail by $[6,12]$ and is not repeated here. In essence, the model is based on solving Parker's transport equation [7] numerically and made applicable for the solar modulation of electrons. This equation is given by:

$$
\frac{\partial f}{\partial t}=-\left(\mathbf{V}+\left\langle\mathbf{v}_{D}\right\rangle\right) \cdot \nabla f+\nabla\left(\mathbf{K}_{s} \cdot \nabla f\right)+\frac{1}{3}(\nabla \cdot \mathbf{V}) \frac{\partial f}{\partial \ln P}+Q .
$$

Here $f(\boldsymbol{r}, P, t)$ is the omni-directional cosmic ray distribution function dependent on vector position $\boldsymbol{r}$, rigidity $P$ and time $t$. The relation between $f$ and differential intensity is simply: $I=$ 
$P^{2} f$. In Eq. 1, $\mathbf{V}$ is the solar wind velocity, and $\mathbf{K}_{s}$ is the full 3D diffusion tensor. It is assumed that $\partial f / \partial t=0$ which means the contribution of short-term modulation effects is neglected with the focus instead on the global spatial and spectral trends. Terms on the right hand side respectively represent convection, gradient and curvature drifts, diffusion and adiabatic energy changes, with $Q$ the JES function. The pitch angle averaged guiding center drift velocity for a near isotropic CR distribution is related to the drift coefficient $K_{D}$ as follows: $\left\langle\mathbf{v}_{D}\right\rangle=\nabla \times\left(K_{D} \mathbf{e}_{B}\right)$ with $\mathbf{e}_{B}=\mathbf{B} / B$ and where $B$ is the magnitude of the averaged background HMF, usually assumed to have a Parkerian geometry. The latter gives very large particle drifts over the polar regions of the heliosphere so that it is a standard practice to modify it in the polar regions of the heliosphere, see e.g. the discussion by [8].

The 3D diffusion tensor consists of three DCs, one parallel and two perpendicular to the background HMF; see also $[1,6,8]$. The spatial dependence of the DCs, assumingly scaling according to $1 / B$, was specified differently over the first $84 \mathrm{AU}$ than in the outer heliosphere, in qualitative agreement with the expectation that the turbulence at and beyond the TS is to increase, with subsequent lower DCs. See also the discussions by [14].

The rigidity dependence of these DCs are also given and discussed by [6]. It is important to note that these DCs are independent of rigidity below $0.4 \mathrm{GV}$, as applicable to electrons.

Only solar minimum conditions is considered with the HCS tilt angle $\alpha=5^{\circ}$. Computations are done for both magnetic polarity epochs, the so-called $\mathrm{A}<0$ and $\mathrm{A}>0$ cycles $[1,8]$. The solar minimum period around 2009 was an A $<0$ cycle. The termination shock (TS) is specified at 94 $\mathrm{AU}$ and the heliopause (HP) at $122 \mathrm{AU}$ to be consistent with V1 observations. Modulation of CRs beyond the HP is not considered [e.g. 9].

\section{Heliopause spectrum and the Jovian source function}

We first briefly discuss the HPS and JES functions which are both modulated according to the assumed modulation parameters. The VLIS for electrons with $E>\sim 30 \mathrm{GeV}$ has always been reasonably well-known from a variety of balloon and space experiments, with the electron spectra from the PAMELA mission [10] as the best recent example. Of course, it is assumed that at these relatively high energies, no solar modulation occurs so that observations at the Earth are considered as a good representation of a VLIS. At lower kinetic energies, $E<500 \mathrm{MeV}$, this was not the case. However, since V1 crossed the HP in August 2012 [2,11], it has been claimed that the VLIS below $100 \mathrm{MeV}$ is actually been observed. First, it became clear that it depicted a power-law shape, similar to the HPS deduced by $[12,13]$. The analysis of these electron observations reported by [2] lead to two possible power-law shapes for the HPS with different spectral indices at these low energies, namely, $E^{1.35}$ and $E^{-1.55}$. For this work only $E^{-1.55}$ is used. A discussion of alternative electron HPS or VLIS is given by [14,15].

The exact power index of the HPS at the low energies does not play a determining role in what we show below. The main aspect in this context is that the HPS has a power-law shape at low energies, making the electron modeling reported here quite interesting. See also the manuscript in this volume on the acceleration of Galactic electrons at the TS. 


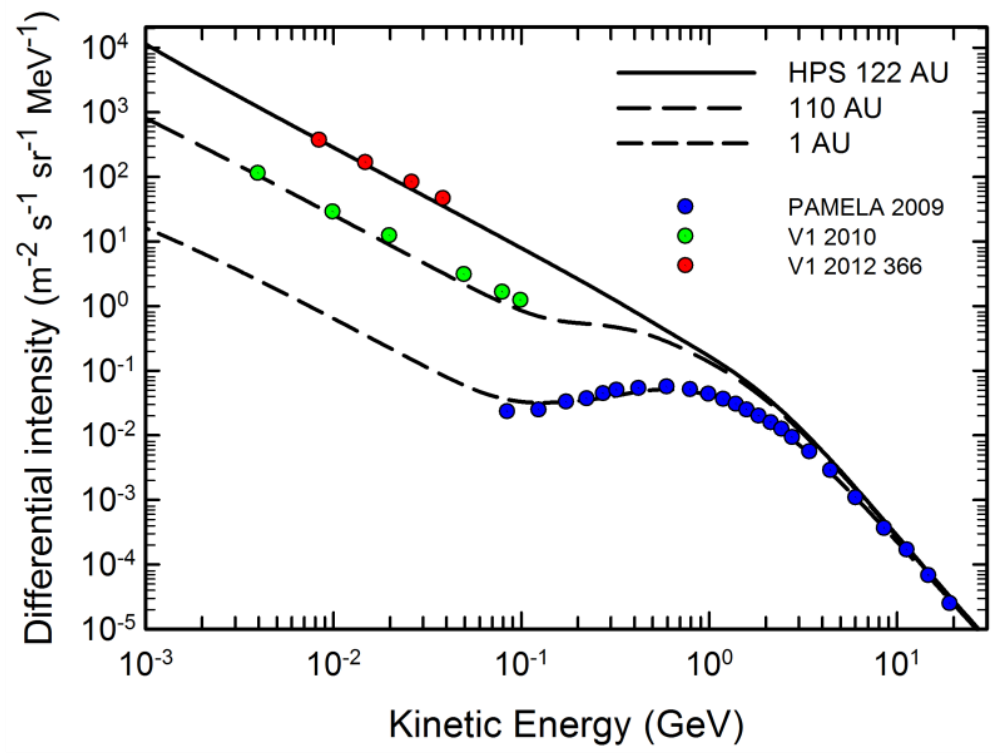

Figure 1: Computed, modulated spectra for Galactic electrons at the Earth (1 AU) and at $110 \mathrm{AU}$, with respect to the HPS at $122 \mathrm{AU}$ as given by Eq. 2, and compared with observations at the Earth from PAMELA [10] during 2009, in the outer heliosphere from V1 (112 AU) during 2010 [e.g. 16], and from V1 on day 366 of 2012 [2].

The HPS for Galactic electrons in terms of differential intensity in units of electrons $\mathrm{m}^{-2} \mathrm{~s}^{-1}$ $\mathrm{sr}^{-1} \mathrm{MeV}^{-1}$ is: For $500 \mathrm{MeV} \leq E \leq 2.0 \mathrm{GeV}$ :

$$
j_{L I S}=\frac{0.14}{\beta^{2}}\left[\frac{\left(E / E_{0}\right)^{1.6}+\left(E_{k 1} / E_{0}\right)^{1.6}}{1+\left(E_{k 1} / E_{0}\right)^{1.6}}\right]^{\frac{b_{1}-a_{1}}{1.6}}\left(E / E_{0}\right)^{a_{1}} \text {, }
$$

and for energies outside this range, it is:

$$
j_{\text {LS }}=\left[4.0 \exp \left(3.0-5.5 \ln (E)-8.0 E_{k 1}^{(-0.7)}\right)\right] \text {. }
$$

Here, $E_{0}=1 \mathrm{GeV}$ to take care of the units, $E_{\mathrm{kl}}=1.4 \mathrm{GeV}, a_{1}=-1.55$ and $b_{1}=-3.22$. This HPS is shown at $122 \mathrm{AU}$ in Fig.1 which will be discussed further below.

The JES function is constructed based mainly on Ulysses [4,5] observations. This source function, again given in units of electrons $\mathrm{m}^{-2} \mathrm{~s}^{-1} \mathrm{sr}^{-1} \mathrm{MeV}^{-1}$ is:

$$
j_{\text {source }}=(300)\left(100 E / E_{0}\right)^{-1.8} e^{\left(-100 E / E_{0}\right)} .
$$

This function is shown in Fig. 2 which is discussed also further below. It has a spectral index of -1.8 for the energy range $2 \mathrm{MeV} \leq E \geq 17 \mathrm{MeV}$, which is consistent with earlier observations [e.g. 18], although quantitatively different from the recent work of [5], who made a critical reevaluation of the relevant Jovian electron observations and what it implies for the JES.

\section{Results and discussion}

The total global modulation of these electrons is illustrated with the main purpose to compare computations for Galactic electrons with Jovian electrons at the Earth in order to determine 


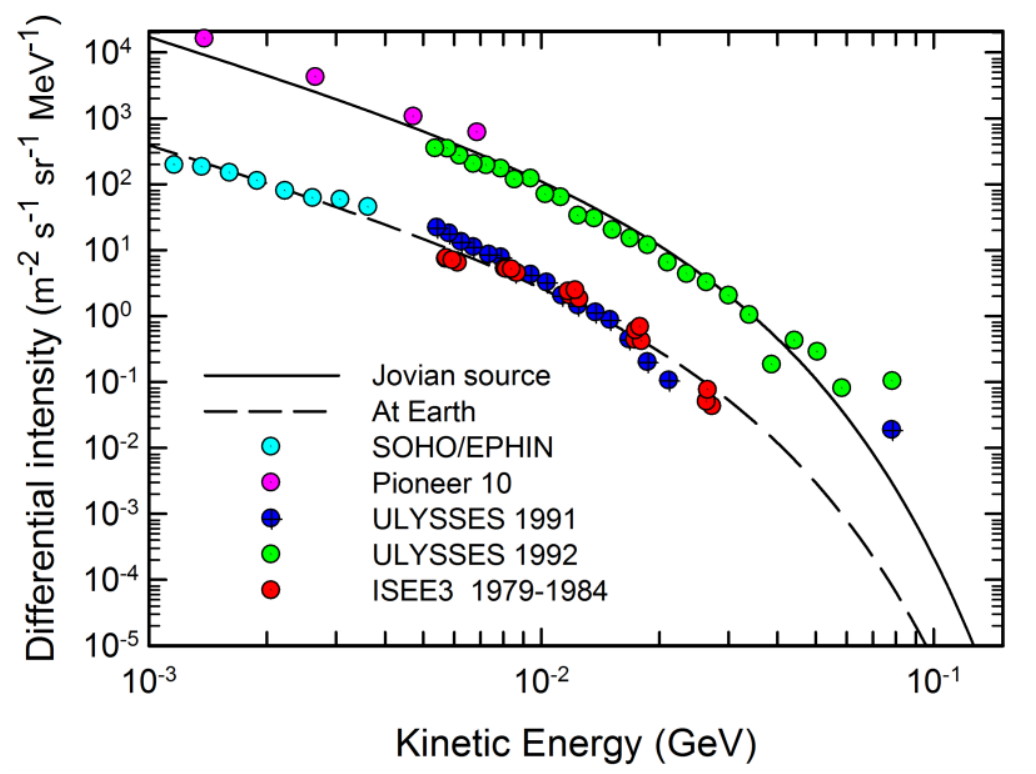

Figure 2: Computed Jovian electron spectrum at the Earth (black dashes) with respect to the JES function (solid black line; as in Eq. 3) at 5 AU, compared to observations from SOHOEPHIN mission [17], Pioneer 10 [18], ULYSSES for 1991 and 1992 [4], and ISEE3 for 19791984 [3]. The JES depicts a power-law shape at low energies but then decrease exponentially above $\sim 15 \mathrm{MeV}$. Above $\sim 30 \mathrm{MeV}$ an additional component seems to be present in the observationa data, which will be dicussed further below.

the energy range where the Jovian electrons dominate over the Galactic component. The intensity that Galactic electrons can have below $50 \mathrm{MeV}$ at Earth is estimated based on this computation. For this procedure, the newly constructed HPS (VLIS) and JES are used in the numerical model as published by [6]. As mentioned, both these source functions are constructed based on relevant CR observations as shown and referenced in Figs. 1 and 2.

Fig. 1 shows the computed Galactic electron spectra at the Earth (1 AU) and at $110 \mathrm{AU}$, with respect to the HPS at 122 AU as given by Eq. 2, and compared with observations as indicated. Evidently, the model, using the constructed HPS to modulate spectra in the outer heliosphere and at the Earth, reproduces the observations well. It is considered as a validation of the modeling approach from $1 \mathrm{MeV}$ up to $30 \mathrm{GeV}$. Note how the spectral slope of the HPS below $\sim 100 \mathrm{MeV}$ is maintained with the modulated spectra up to the Earth because the DCs for these electrons are independent of energy; see also $[1,6,12,13]$. Also note from the computed spectrum at $110 \mathrm{AU}$ how significant Galactic electron modulation is inside the heliosheath.

Fig. 2 shows the JES function at $5 \mathrm{AU}$ and the corresponding computed modulated spectrum at the Earth (1 AU), together with observations as indicated; data from Ulysses were for 1991 when it was close to the Earth, and for 1992 when it was close to Jupiter (5 AU). For Pioneer 10, the data [18] were averaged over four periods when the spacecraft was in the Jovian magnetosphere; the ISEE3 observations [3, see also 5] are for the period 1979 to 1984.

It follows from this figure that below $\sim 15 \mathrm{MeV}$, the power-law shape of the JES dominates, but above this energy the intensity decreases rapidly so that the Jovian electrons subside quickly. It is evident that at Earth the model results are compatible with the observations down 
to very low energies. Note that also in this case the spectral shape of the JES at low energies is maintained by the modulated spectrum up to the Earth. According to the observational data these Jovian electrons evidently are observed up to 20 to $30 \mathrm{MeV}$ at the Earth, but above this energy range a change in the observed spectral slope becomes evident which appears more in line with what is expected of Galactic electrons. This aspect is discussed further with the next figure.

Fig. 3 shows the combined modulation of Jovian and Galactic electrons computed with respect to the constructed HPS and the JES. The modulated Galactic spectra are shown at the Earth (1 AU) and at $110 \mathrm{AU}$, with the HPS at $122 \mathrm{AU}$. The modulated Jovian spectrum is also shown at the Earth with respect to the JES at 5 AU. As the main result of this modeling, the combined Jovian and Galactic electron spectrum at the Earth is shown as the solid red line, from $1 \mathrm{MeV}$ to $30 \mathrm{GeV}$. Evidently, the Jovian electron spectrum differs significantly from that of the

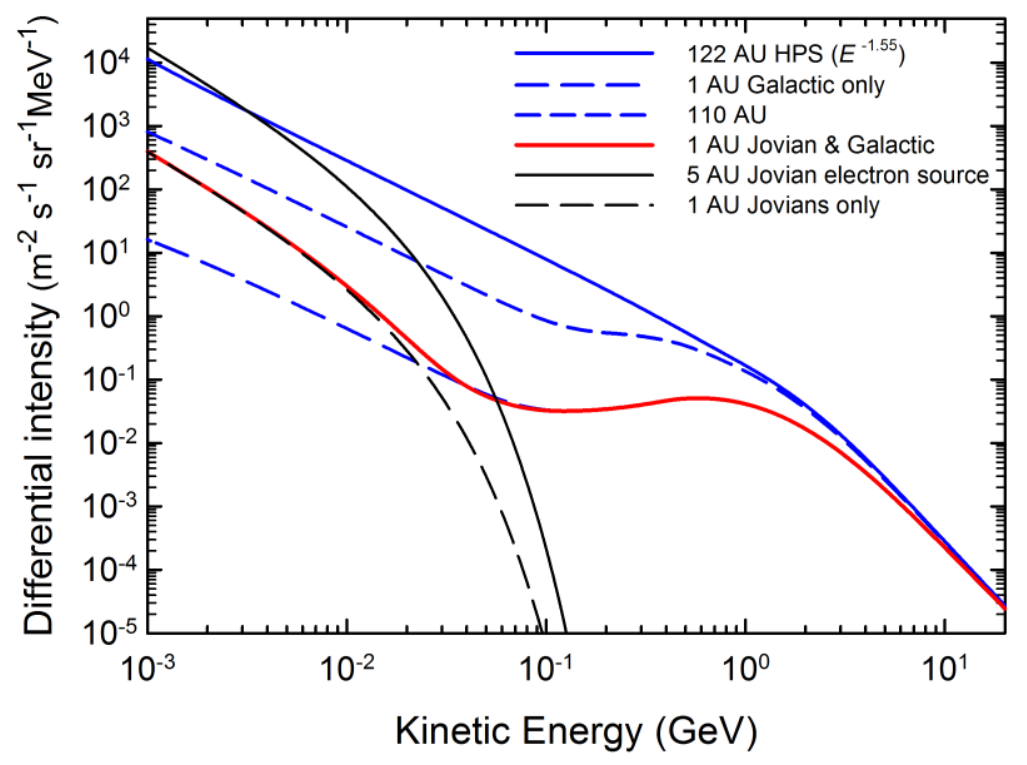

Figure 3: Combined Galactic and Jovian electron spectra computed as a function of kinetic energy. Unmodulated HPS $\left(E^{-1.55}\right)$ for Galactic electrons is shown at 122 AU (blue solid line) with modulated spectra at $110 \mathrm{AU}$ (blue short-dashes) and at the Earth (1 AU; blue longdashes). Jovian source function is shown at 5 AU (black solid line) with its modulated spectrum at the Earth (1 AU; black long-dashes). The modulated, combined Galactic and Jovian spectrum is shown at the Earth (1 AU; red solid line) from $1 \mathrm{MeV}$ to $30 \mathrm{GeV}$.

Galactic electrons. This emphasizes the difference in the characteristical forms (shapes) of modulated Jovian and Galactic electrons, especially at the Earth. Notice that below $\sim 5 \mathrm{MeV}$ the HPS and JES are surprisingly close in terms of differential intensities.

It follows from these results that at the Earth, the Jovian electrons dominate the electron spectrum below $\sim 15 \mathrm{MeV}$ but as the Jovian spectrum sharply decreases with increasing energy, the shape of the Galactic electron spectrum begins to dominate. In the energy range $15 \mathrm{MeV} \geq E$ $\leq 40 \mathrm{MeV}$ the spectrum is still a mixture of Galactic and Jovian electron but above this range, the spectral shape of the Galactic electron is dictating. Higher up in energy the characteristic shape of the modulated Galactic electron spectrum takes over, as determined by the interplay between diffusion and drifts [see also 14]. 
Based on the blue long-dashed line in Fig. 3, the intensity that the Galactic electron can have at $1 \mathrm{MeV}$, for example, is $\sim 20$ electrons $\mathrm{m}^{-2} \mathrm{~s}^{-1} \mathrm{sr}^{-1} \mathrm{MeV}^{-1}$, whereas the Jovian electrons have an intensity of $\sim 400$ electrons $\mathrm{m}^{-2} \mathrm{~s}^{-1} \mathrm{sr}^{-1} \mathrm{MeV}^{-1}$.

It was shown by [12] that the Jovian electrons are dominantly present in the heliosphere up to 20 AU but drops off significantly beyond this value. This has been recalculated and illustrated in Fig. 4, for $6 \mathrm{MeV}$ and $20 \mathrm{MeV}$ Jovian and Galactic electrons, respectively.

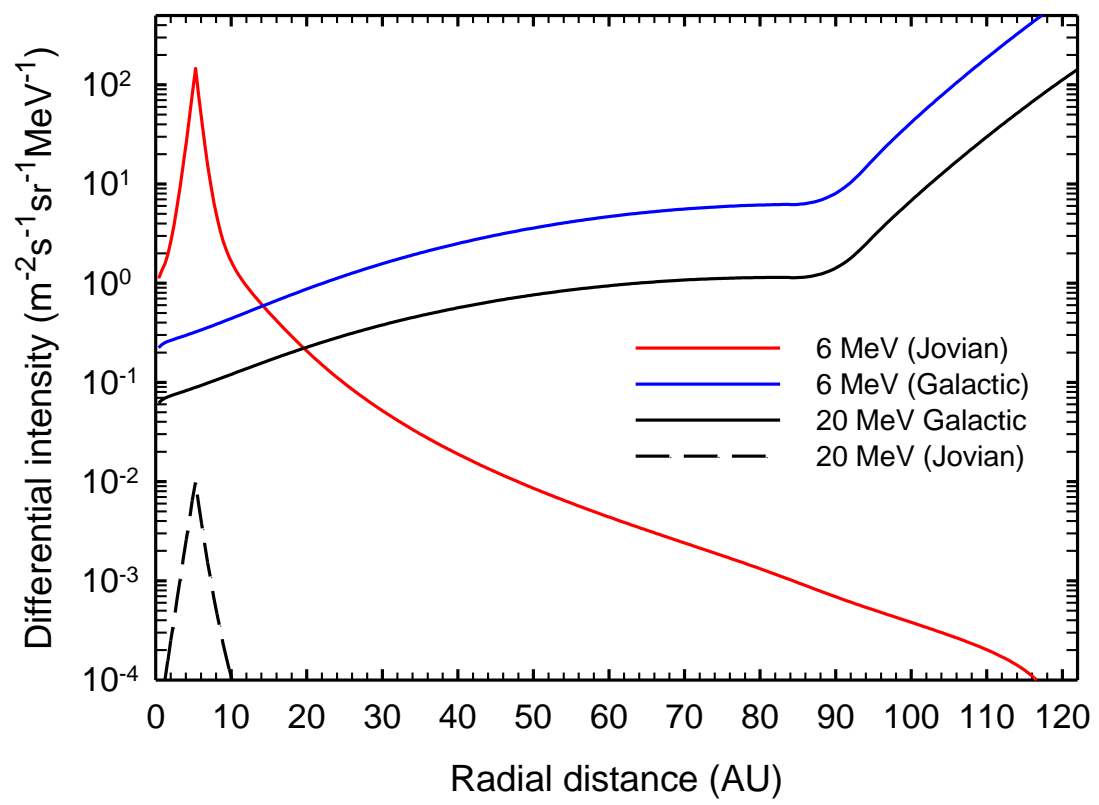

Figure 4: Computed radial profiles of $6 \mathrm{MeV}$ and $20 \mathrm{MeV}$ Jovian and Galactic electron intensities as indicated, from the Earth up to the HP at $122 \mathrm{AU}$. Intensities are computed with respect to the JES and HPS shown in Figs. 1 and 2. The TS is at 94 AU from where the Galactic electrons pick up sharply to reach the values of the HPS at these energies at the HP. Note how significant the drop in Jovian electrons is from 6 to $20 \mathrm{MeV}$, but less so for Galactic electrons.

\section{Discussion and Conclusions}

In view of the facts that the low-energy electron observations are complex, even controversial, and that solar modulation may influence what the JES function might be, this function remains contentious, as is illustrated and discussed by [5]. As such it is difficult to say with certainty exactly where the Jovian electrons dominate in the heliosphere. Obviously, the choice of the JES function, the HPS and the choice of DCs influence the total modulation that occurs between the Earth and the heliospheric boundary.

Observationally, the Galactic electron intensity below $\sim 50 \mathrm{MeV}$ is not known at Earth because of the dominance of the Jovian electrons. From this study, we find that the Jovian electrons dominate the electron spectrum below $\sim 15 \mathrm{MeV}$ but as the Jovian spectrum sharply decreases with increasing energy, the shape of the Galactic electron spectrum begins to dominate. In the energy range $15 \mathrm{MeV} \geq E \leq 40 \mathrm{MeV}$ the spectrum is a mixture of Galactic and Jovian electron but above this range, the spectral shape of the Galactic electron is dominant. Higher up in energy the characteristic shape of the modulated Galactic electron spectrum takes over.

We conclude that the highest differential intensity for Galactic electrons at Earth for $1 \mathrm{MeV}$ is $\sim 20 \mathrm{~m}^{-2} \mathrm{~s}^{-1} \mathrm{sr}^{-1} \mathrm{MeV}^{-1}$ whereas for Jovian electrons it is $\sim 400 \mathrm{~m}^{-2} \mathrm{~s}^{-1} \mathrm{sr}^{-1} \mathrm{MeV}^{-1}$. 
MSP acknowledges the partial financial support of the South African National Research Foundation (NRF), also Bernd Heber and Adrian Vogt for many discussions on Jovian electrons, and Bill Webber for interesting discussions on the V1 electron data. RRN acknowledges the NRF's and SANSA's contributions to her PhD bursary.

\section{References}

[1] M.S. Potgieter. Solar modulation of cosmic rays. Living Rev. Solar Phys. 10, 3, 2013.

[2] E.C. Stone, A.C. Cummings, F.B. McDonald et al. Voyager 1 observes low-energy galactic cosmic rays in a region depleted of heliospheric ions, Science 341, 150, 2013.

[3] D. Moses, Jovian electrons at 1 AU, 1978-1984, ApJ. 313, 471, 1987.

[4] B. Heber, A. Kopp, H. Fichtner, S.E.S. Ferreira. On the determination of energy spectra of MeV electrons by the Ulysses COSPIN/KET. Adv. Space Res. 35, 605, 2005.

[5] A. Vogt, B. Heber, A. Kopp, et al. Jovian electrons in the inner heliosphere: Proposing a new source spectrum based on 30 years of measurements, submitted to A\&A, 2017.

[6] R.R. Nndanganeni, M.S. Potgieter. The energy range of drift effects in the solar modulation of cosmic ray electrons. Adv. Space Res. 58, 453, 2016.

[7] E.N. Parker, The passage of energetic charged particles through interplanetary space, Planet. Space Sci. 13, 9, 1965.

[8] J.L. Raath, M.S. Potgieter, R. D. Strauss, A. Kopp. The effects of magnetic field modifications on the solar modulation of cosmic rays with a SDE-based model. Adv. Space Res. 57, 1965, 2016.

[9] Xi Lou, M. Zhang, M.S. Potgieter et al., A numerical simulation of cosmic-ray modulation near the heliopause, ApJ. 808, 82, 2015.

[10] O. Adriani, et al. Time dependence of the e- flux measured by PAMELA during the July 2006- December 2009 solar minimum, ApJ. 810, 142, 2015.

[11] D.A. Gurnett, W.S. Kurth, L.F. Burlaga, N.F. Ness. In situ observations of interstellar plasma with Voyager 1, Science 341, 1489, 2013.

[12] M.S. Potgieter, R.R. Nndanganeni. The solar modulation of electrons in the heliosphere. Astrophys. Space Sci. 345, 33, 2013.

[13] M.S. Potgieter, R.R. Nndanganeni. A local interstellar spectrum for galactic electrons, Astropart. Phys. 48, 25, 2013.

[14] M.S. Potgieter, E.E. Vos. Difference in the heliospheric modulation of cosmic-ray protons and electrons during solar minimum of 2006 to 2009. A\&A. 601, A23, 2017.

[15] D. Bisschoff, M.S., Potgieter. Implications of Voyager 1 observations beyond the heliopause for the local interstellar electron spectrum, ApJ. 749, 166, 2014.

[16] R.A. Caballero-Lopez, H. Moraal, F.B. McDonald. The modulation of galactic electrons in the heliosheath, ApJ. 725, 121, 2010.

[17] P. Kühl, N. Dresing, P. Dunzlaff, et al., Spectrum of galactic and Jovian electrons, Proc. $33^{\text {rd }}$ Int. Cosmic Ray Conf. (Rio de Janeiro, Brazil), icrc2013-0072, 2013.

[18] C. Lopate. Jovian and galactic electrons $(2-30 \mathrm{MeV})$ in the heliosphere from 1 to $50 \mathrm{AU}$, Proc. $22^{\text {nd }}$ Int. Cosmic Ray Conf. (Dublin, Ireland), 2, 149, 1991. 